\title{
Current-induced dynamical tilting of chiral domain walls in curved microwires
}

\author{
Cite as: Appl. Phys. Lett. 116, 182404 (2020); doi: 10.1063/5.0005186 \\ Submitted: 18 February 2020 . Accepted: 21 April 2020 . \\ Published Online: 6 May 2020
}
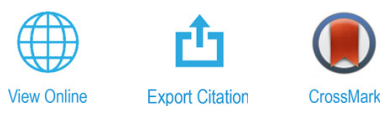

\begin{abstract}
Simone Finizio, ${ }^{1, a)}$ (ID Sebastian Wintz, ${ }^{2}$ Sina Mayr, ${ }^{1,3}$ Alexandra J. Huxtable, ${ }^{4}$ (D) Manuel Langer, Joe Bailey, ${ }^{1,5}$ Gavin Burnell, ${ }^{4}$ (D) Christopher H. Marrows, ${ }^{4}$ (D) and Jörg Raabe ${ }^{7}$ (iD
\end{abstract}

\author{
AFFILIATIONS \\ ${ }^{7}$ Paul Scherrer Institut, 5232 Villigen PSI, Switzerland \\ ${ }^{2}$ Max-Planck-Institut für Intelligente Systeme, 70569 Stuttgart, Germany \\ ${ }^{3}$ Laboratory for Mesoscopic Systems, Department of Materials, ETH Zurich, 8093 Zurich, Switzerland \\ ${ }^{4}$ School of Physics and Astronomy, University of Leeds, Leeds LS2 9JT, United Kingdom \\ ${ }^{5}$ École Polytechnique Fédérale de Lausanne (EPFL), 1015 Lausanne, Switzerland
}

${ }^{a)}$ Author to whom correspondence should be addressed: simone.finizio@psi.ch

\begin{abstract}
We report on the investigation of current-induced domain wall motion of Néel domain walls in perpendicularly magnetized microwires with curved geometries in the flow regime. The investigation was performed by time-resolved scanning transmission $\mathrm{x}$-ray microscopy. In particular, we studied the dynamical tilting of the Néel domain walls, observing that an asymmetric behavior in the domain wall tilt appears upon an inversion of the polarity of the current pulse driving the motion, an effect not predicted by state-of-the-art theories and micromagnetic modeling.
\end{abstract}

Published under license by AIP Publishing. https://doi.org/10.1063/5.0005186

The motion of magnetic domains and domain walls (DWs) can be excited using electrical currents (current-induced domain wall motion-CIDWM), exploiting effects such as spin transfer torques ${ }^{1}$ and spin-orbit torques (SOTs). ${ }^{2,3}$ SOT-driven CIDWM has recently allowed for the observation of time-averaged DW velocities up to a kilometer per second. ${ }^{4,5}$ These large DW velocities were observed in multilayer stacks engineered to exhibit a combination of perpendicular magnetic anisotropy (PMA) and Dzyaloshinskii-Moriya (DM) interaction, which allows for the stabilization of perpendicularly magnetized domains with a defined DW chirality.

The most advertised application for CIDWM is in shift-register devices such as the racetrack memory. ${ }^{3,6}$ Here, multiple works have demonstrated the feasibility of the racetrack memory design both quasi-statically $^{7-9}$ and dynamically. ${ }^{10,11}$ Furthermore, various geometrical designs of the racetrack enable different operations on the DWs, including DW logic ${ }^{12}$ and DW sorting, ${ }^{13}$ providing an avenue toward applications such as DW computing.

The applications mentioned above rely on non-trivial geometries of magnetic microwires, in particular, involving the use of curved sections. However, recent experimental investigations ${ }^{4}$ have shown that the behavior of the CIDWM process in curved geometries cannot be directly extrapolated from the CIDWM behavior in straight wires. In particular, the curvature of the microwires appears to dramatically influence the CIDWM process. To interpret the experimental results, obtained with quasi-static Kerr-effect microscopy measurements, Garg and co-authors ${ }^{4}$ have developed an extension of the $1 \mathrm{D}$ model, ${ }^{7}$ referred to as the $2 \mathrm{D}$ model, to describe the influence of the microwire curvature on the CIDWM process. This model was employed, corroborated by micromagnetic simulations, to predict the dynamical behavior of DWs in a curved microwire and, especially, the dynamical variations in their velocity and tilting. In particular, the model predicts a different average DW velocity and dynamical DW tilting depending on both the sign of the curvature of the microwire (i.e., a convex or concave microwire with respect to the current flow) and the local magnetic configuration (i.e., whether an up-down or down-up domain with respect to the current flow is present). These predictions are employed to interpret the different velocities of the two DW types observed in quasi-static experiments. ${ }^{4}$ The prediction of a substantial difference in the dynamical DW behavior of curved microwires, depending on the domain wall type and on the curvature of the microwire, provides a strong motivation to perform a pump-probe experimental investigation where the CIDWM dynamics in curved microwires are directly imaged. 
Using pump-probe time-resolved scanning transmission $\mathrm{x}$-ray microscopy (TR-STXM) imaging, ${ }^{14}$ it is possible to investigate the dynamics of the CIDWM process at the nanoscale (spatial resolution on the order of $25 \mathrm{~nm}$ ) with a probing time step down to 100-200 ps (time resolution of $70 \mathrm{ps}$ ). ${ }^{10} \mathrm{We}$, therefore, made use of these developments in TR-STXM imaging to investigate the dynamical behavior of Néel DWs during their current-induced displacement in curved microwires. We employed multilayered $\mathrm{Pt} / \mathrm{Co}_{68} \mathrm{~B}_{32} / \mathrm{Ir}$ stacks for these experiments, for which an asymmetric behavior in the DW tilting when injecting current pulses of opposite polarities could be observed, a behavior not expected from theoretical modeling.

The investigation of the dynamical tilting of chiral Néel DWs was carried out on a microwire fabricated out of a $\mathrm{Ta}(3 \mathrm{~nm}) / \mathrm{Pt}(3.4 \mathrm{~nm}) /$ $\left[\mathrm{Co}_{68} \mathrm{~B}_{32}(0.9 \mathrm{~nm}) / \mathrm{Ir}(0.8 \mathrm{~nm}) / \mathrm{Pt}(1.8 \mathrm{~nm})\right] \times 3 / \mathrm{Pt}(2.6 \mathrm{~nm})$ multi-layer stack (from now on referred to as $\mathrm{Pt} / \mathrm{CoB} / \mathrm{Ir}$ ) exhibiting both PMA and interfacial DM interaction, which leads to the stabilization of chiral Néel DWs between perpendicularly magnetized domains. ${ }^{10}$ The microwire was patterned by electron beam lithography on top of a $200 \mathrm{~nm}$ thick $\mathrm{x}$-ray transparent $\mathrm{Si}_{3} \mathrm{~N}_{4}$ membrane on a high-resistivity intrinsic $\mathrm{Si}$ support frame. The microwire was fabricated in a curved geometry with a width $w$ of $1 \mu \mathrm{m}$ and a radius of curvature $R_{\mathrm{C}}$ of $4 \mu \mathrm{m}$. In order to inject current pulses across the microwire, $200 \mathrm{~nm}$ thick $\mathrm{Cu}$ contacts were patterned on the two ends of the microwire. To nucleate a domain wall at the center of the curved section of the microwire, a $400 \mathrm{~nm}$ thick $\mathrm{Cu}$ Oersted line was fabricated on top of the microwire, using a $100 \mathrm{~nm}$ thick $\mathrm{SiO}_{2}$ layer to guarantee the electrical insulation between the microwire and the Oersted line. A scanning electron micrograph of the patterned sample, along with an overview of the electrical and timing connections employed for the TR-STXM measurements reported here, is shown in Fig. 1(a). The non-uniform spatial distribution of the current density in the curved microwire, simulated using the commercial finite element simulator ANSYS, is shown in Fig. 1(b). More details about the fabrication of similar samples, the electrical connections employed for the TR-STXM measurements, and the micromagnetic parameters of the Pt/ $\mathrm{CoB} / \mathrm{Ir}$ multilayer stack are given in Ref. 10 and in the supplementary material. (a)

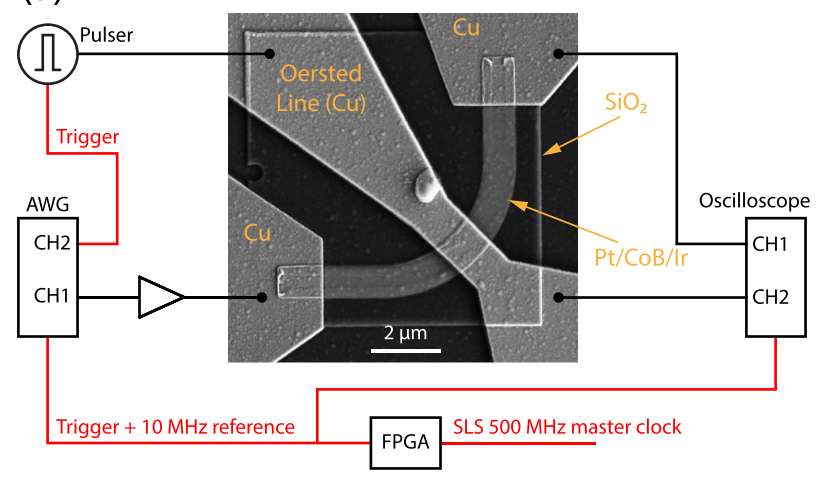

(b)

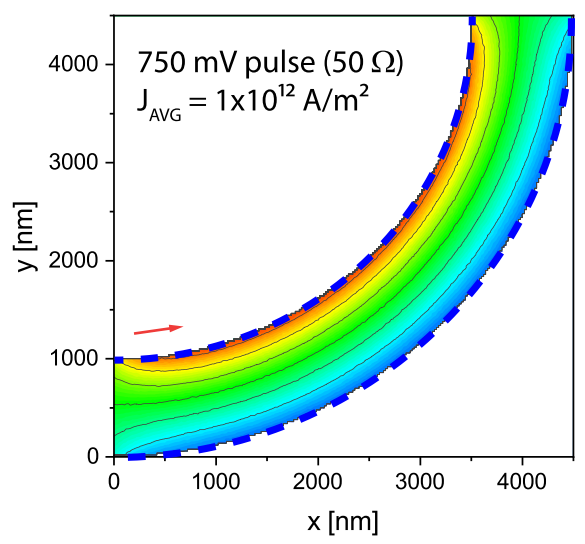

(d)

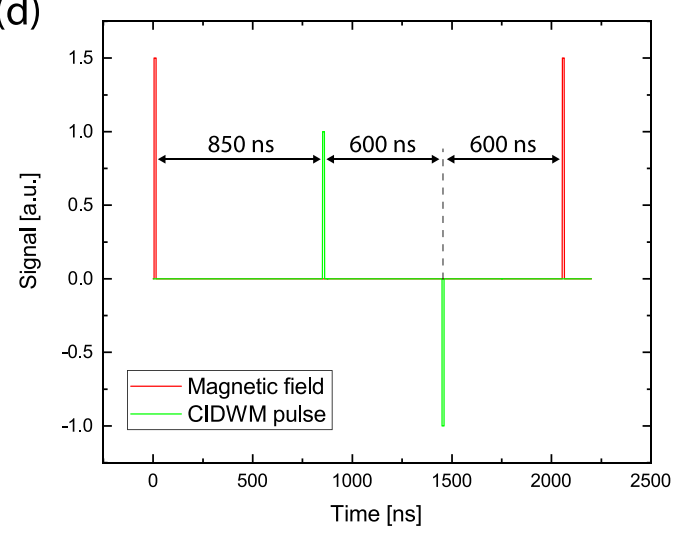

FIG. 1. (a) Scanning electron micrograph of one of the $1 \mu \mathrm{m}$ wide curved Pt/CoB//r microwires investigated here. The Oersted line is electrically insulated from the Pt/CoB/lr microwire by a $100 \mathrm{~nm}$ thick $\mathrm{SiO}_{2}$ film. A schematic overview of the electrical (black) and timing (red) connections is also shown. (b) Finite-element simulation of the current density in the curved microwire upon the injection of a $750 \mathrm{mV}$ pulse (over a termination of $50 \Omega$ ), resulting in an average current density of $1 \times 10^{12} \mathrm{~A} \mathrm{~m}^{-2}$. The non-uniform distribution of the current density in the curved microwire can be observed. The blue dashed line marks the edges of the microwire, while the red arrow indicates the direction of the injected current pulse in the simulation. (c) Quasi-static STXM image of the magnetic configuration of the curved Pt/CoB/lr microwire after its initialization through the injection of a current pulse across the Oersted line. The legend at the bottom of the image depicts the direction of the magnetization corresponding to the contrast in the image, and the orange arrows in the image depict the chirality of the Néel domain wall by showing the orientation of the in-plane spins in the domain wall. (d) Schematic time trace of the magnetic field and CIDWM pulses employed over one cycle of excitation (about $2 \mu \mathrm{s}$ ). 
The TR-STXM experiments were performed at the SurfaceInterface Microscopy twin-undulator beamline (X11MA) of the Swiss Light Source, ${ }^{15}$ employing a prototype ptychography endstation ${ }^{16}$ operated with a point detector instead of a $2 \mathrm{D}$ detector (i.e., in the STXM mode). Prior to time-resolved imaging, the Pt/CoB/Ir microwire was initialized in a bi-domain state (with a Néel DW separating the two domains) by injecting a $4 \mathrm{~ns}$ long, $350 \mathrm{~mA}$ current pulse across the Oersted line, causing the nucleation of the two domains from a uniformly magnetized configuration [see Fig. 1(c)—the orange arrows indicate the orientation of the in-plane magnetization in the Néel domain wall, according to the calculations presented in Ref. 10]. The TR measurements were then performed in the remnant state. The excitation signal consisted of two current pulses of equal width and amplitude, but opposite polarities, injected across the $\mathrm{Pt} / \mathrm{CoB} / \mathrm{Ir}$ microwire, both causing a displacement of the DW in opposite directions. To allow for the complete dissipation of the heat generated by the injection of the current pulse (see Ref. 17 for a measurement of the heating caused by the injection of a current pulse across a $\mathrm{Pt} / \mathrm{CoB} / \mathrm{Ir}$ microwire), as well as the complete relaxation of any dynamical processes, the two CIDWM pulses were separated by $600 \mathrm{~ns}$. To guarantee the reproducibility of the dynamical processes necessary for pumpprobe imaging, the original magnetic configuration in the $\mathrm{Pt} / \mathrm{CoB} / \mathrm{Ir}$ microwire was restored by the injection of a current pulse across the Oersted line. The reset pulse was injected $600 \mathrm{~ns}$ after the second CIDWM pulse. The first CIDWM pulse is injected $850 \mathrm{~ns}$ after the reset pulse, resulting in a width of about $2 \mu$ s for one cycle of excitation. A schematic time trace of the CIDWM and magnetic field pulses is shown in Fig. 1(d). CIDWM pulse widths between 10 and $20 \mathrm{~ns}$ and spatially averaged current densities up to $7.2 \times 10^{11} \mathrm{~A} \mathrm{~m}^{-2}$ were employed for the experiments presented here. These pulse widths were selected to be able to observe a tilting of the DW, which was not observed for shorter pulse widths in this material. ${ }^{10}$ In order to acquire sufficient statistics for a time-resolved image with the pump-probe imaging protocol, the excitation of the sample needs to be repeated on the order of $10^{9}-10^{10}$ times, providing, therefore, a strong support toward the reproducibility of the dynamical processes. We furthermore investigated a number of different microwires with the same geometry, to guarantee that potential growth or patterning imperfections of single samples did not affect the results we report here.

The TR-STXM images were acquired employing circularly polarized $\mathrm{x}$-rays tuned to the Co $\mathrm{L}_{3}$ absorption edge. From these images, shown in the supplementary material, we calculated the tilting of the DW during and after the application of the current pulses causing the CIDWM (the details on the calculation of the DW tilting angle are given in the supplementary material). Due to the requirement of long integration times caused by the $2 \mu$ s long excitation cycle, most of the TR measurements were performed with a time step of $2 \mathrm{~ns}$. For the time series of highest interest, more detailed TR measurements employing a time step of $400 \mathrm{ps}$ were performed.

Examples of the calculated time traces of the DW tilt during the CIDWM process are shown in Figs. 2(a) and 2(b). From these time traces, a strong difference in the dynamical tilting of the DW can be observed upon the inversion of the CIDWM pulse polarity. In particular, the injection of a positive current pulse [see Fig. 2(c) for the sign convention for the current pulses] leads to a dynamical DW tilting of several degrees, while the injection of a negative current pulse does not lead to a detectable DW tilting. These results were obtained for a down-up domain configuration [see Fig. 2(c)]. Inverting the domain configuration (i.e., up-down domain configuration in this case) leads to an inversion of tilting: a negative current pulse was observed to lead to a detectable DW tilting, while a positive current pulse will, instead, not cause a detectable DW tilting.

While the domain wall accelerates almost instantaneously to its final velocity, ${ }^{10}$ the tilting of the DW follows instead a longer and more complex dynamical process. We, therefore, investigated the dynamical behavior of the DW tilting using a smaller time step (400 ps) for the TR-STXM images. This was carried out for the positive
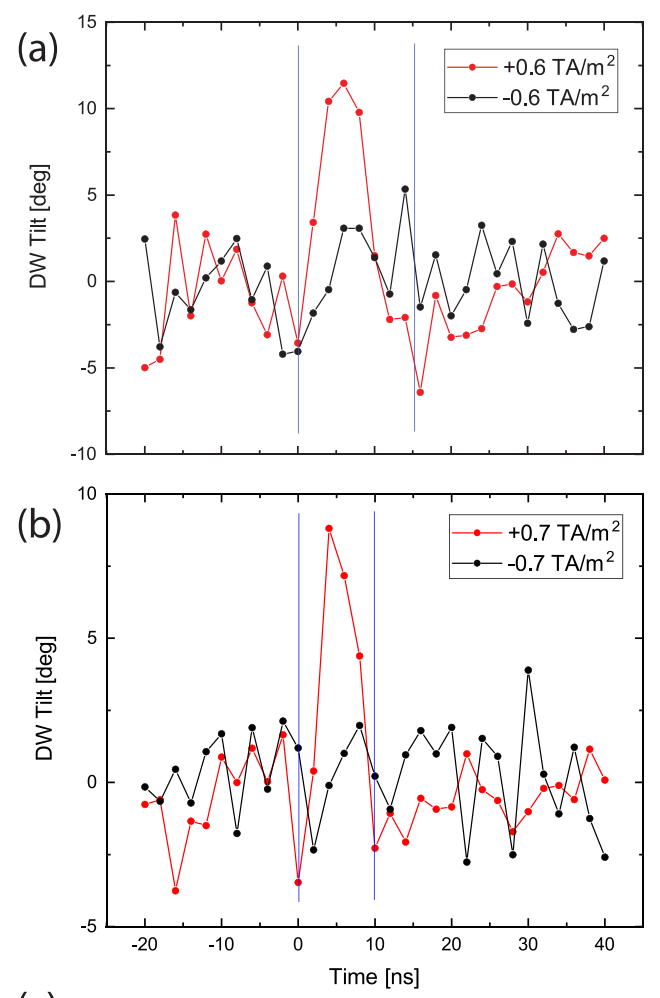

(c)

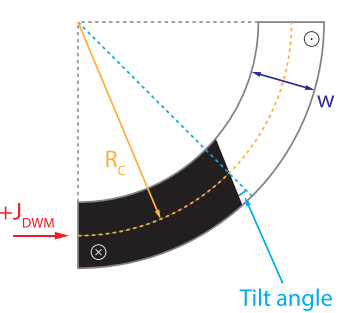

FIG. 2. Time traces ( 2 ns time step) depicting the chiral DW tilting angle during the CIDWM in the Pt/CoB/lr microwire when applying current pulses of (a) $15 \mathrm{~ns}$, with a spatially averaged current density of $6 \times 10^{11} \mathrm{~A} \mathrm{~m}^{-2}$ and of (b) $10 \mathrm{~ns}$, with a spatially averaged current density of $7.2 \times 10^{11} \mathrm{~A} \mathrm{~m}^{-2}$. A clear difference in the DW tilting when inverting the pulse polarity can be observed. The vertical cyan lines mark the time interval during which the current pulse is applied. The convention for the calculation of the DW angle is shown in (c), along with the definition of the radius of curvature $R_{\mathrm{C}}$ and the width $w$ of the $\mathrm{Pt} / \mathrm{CoB} / \mathrm{lr}$ microwire. The sign convention for the polarity of the CIDWM pulse is depicted in red in (c), where the conventional current direction is indicated. The domain configuration shown in (c) is defined as down-up in this work. 
CIDWM reported in Fig. 2(a), as shown in Fig. 3. From this figure, it is possible to observe that the tilting of the DW begins synchronously with the CIDWM pulse, reaches its maximum tilt of about $13^{\circ} \pm 3^{\circ}$ in about $8 \mathrm{~ns}$, and then tilts back to almost $0^{\circ}$ in the remainder of the CIDWM pulse. Another observation that can be made from the time trace shown in Fig. 3 is that, after the end of the CIDWM pulse, the DW exhibits a small negative tilting $\left(-5^{\circ} \pm 3^{\circ}\right)$, which then recovers back to $0^{\circ}$ in about $15 \mathrm{~ns}$. This, together with the progressive increase in the DW tilt when the CIDWM pulse is first applied, indicates that the tilting of the DW occurs over longer timescales than the changes in its velocity, as we inferred in our previous work. ${ }^{10}$

A first possible interpretation of the observed changes in the DW tilting angle when inverting the polarity of the current pulses could be that the current pulse affects the spin configuration of the DW, which would alter its response to a second current pulse (in the experiments reported here, we always injected a positive current pulse first, followed by a negative current pulse). However, given the fact that the behavior of the DW tilting inverts (i.e., a tilting is observed for the negative, but not for the positive pulse) when inverting the domain orientation, we can reliably assert that the current pulse does not appear to cause significant changes in the spin configuration of the DW.

The substantial changes in the dynamical tilting angle of the DW when inverting the polarity of the CIDWM pulse are explained neither by the inhomogeneous distribution of the current density in the curved microwire nor by the Oersted fields generated by the injection of the current pulse. This can be explained from symmetry considerations: an inversion in the sign of the current pulse would lead to an inversion of the sign of the current density, but not of its magnitude. This would then lead to an inversion of the sign of the DW tilting and not to its absence. Similarly, an inversion in the sign of the current pulse would lead to an inversion in the sign of the Oersted field generated, which should give rise to an inversion in the sign of the DW tilting. Therefore, the explanation for this behavior could originate from the interaction with the SOTs generated by the Pt layers that constitute the multilayered superlattice we investigated here. In contrast to the multilayered stack investigated in Ref. 4, where only a single heavy metal layer generates the SOTs that displace the DW, the $\mathrm{Pt} / \mathrm{CoB} / \mathrm{Ir}$ stack investigated here contains four different Pt layers that can contribute to

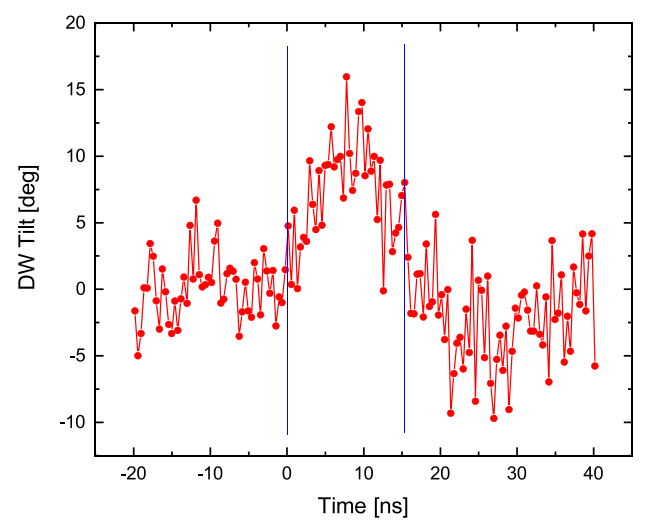

FIG. 3. Time trace (400 ps time step) depicting the DW tilting during the application of a positive current pulse ( $15 \mathrm{~ns}$ long) with a spatially averaged current density of +6 $\times 10^{11} \mathrm{~A} \mathrm{~m}^{-2}$. A down-up domain configuration was initialized for this measurement. The vertical cyan lines mark the time interval during which the current pulse is applied. the SOTs generated by the injection of the pulse. These can, in principle, interact in complex ways with the three CoB layers, leading to nontrivial effects such as the asymmetries in the DW tilting we observed.

According to the micromagnetic and theoretical modeling presented in Ref. 4, we should expect that the average DW velocity should substantially change when inverting the polarity of the CIDWM pulse, given the major difference in the dynamical tilting of the DW between the two cases. However, we did not observe any meaningful changes in the DW velocity when inverting the current pulse (the DW velocity was measured to be about $40 \mathrm{~m} \mathrm{~s}^{-1}$ when injecting a pulse of $7.2 \times$ $10^{11} \mathrm{~A} \mathrm{~m}^{-2}$ ). This does not follow the predictions of the 2D model, as otherwise the observed DW tilt should have exhibited the same magnitude when inverting the polarity of the CIDWM pulse, independent from either the sign of the DM interaction or the SOTs.

In conclusion, we have investigated the dynamical tilting of chiral Néel DWs in curved Pt/CoB/Ir microwires during their CIDWM using TR-STXM imaging. We have observed an asymmetric behavior of the DW tilting upon changing the sign of the injected current pulses, which is, however, not reflected in a change in the DW velocity (i.e., the DW velocity remains unchanged when inverting the sign of the injected current pulse). Such observation of a considerable change in the DW tilting, but not in the DW velocity, does not follow the expected behavior for the CIDWM processes in curved microwires predicted from micromagnetic models. The physical origin behind the observed asymmetric behavior of the DW tilting could be due to the interaction between the SOTs generated by the multiple Pt layers comprising the $\mathrm{Pt} / \mathrm{CoB} / \mathrm{Ir}$ multilayer stack and the magnetic CoB layers. Further studies are, therefore, necessary to completely understand the influence of the SOTs and of the geometrical curvature on the dynamical behavior of the DW during its CIDWM in curved microwires. This asymmetric behavior in the dynamical DW tilting with respect to the polarity of the CIDWM pulse could find applications in the design of DW-based logic or sorting devices. Here, the duration and polarity of the CIDWM pulse could be employed to define the logic operation, providing an additional arrow in the quiver for DW-based devices, extending, in principle, the range of operations beyond the results already reported for straight microwires in Ref. 13.

See the supplementary material for the following: CIDWM_SI.pdf: The magnetometry characterization of the $\mathrm{Pt} / \mathrm{CoB} / \mathrm{Ir}$ multilayer stack investigated here, The analysis method used to extract the DW tilting angle from the TR-STXM images, and the comparison between the observed DW tilting for our experiments vs the DW tilting calculated in Ref. 4; 6E11_POS_2ns.avi-Video1: Time-resolved STXM image (video) showing the CIDWM under the application of $\mathrm{a}+610^{11} \mathrm{~A} \mathrm{~m}^{-2}$ current pulse with a time step of 2 ns [Fig. 2(a)]; 6E11_NEG_2ns.avi-Video2: Time-resolved STXM image (video) showing the CIDWM under the application of a $-6 \times 10^{11} \mathrm{~A} \mathrm{~m}^{-2}$ current pulse with a time step of 2 ns [Fig. 2(a)]; 7E11_POS_2ns.aviVideo3: Time-resolved STXM image (video) showing the CIDWM under the application of a $+7.2 \times 10^{11} \mathrm{~A} \mathrm{~m}^{-2}$ current pulse with a time step of 2 ns [Fig. 2(b)]; 7E11_NEG_2ns.avi-Video4: Timeresolved STXM image (video) showing the CIDWM under the application of a $-7.2 \times 10^{11} \mathrm{~A} \mathrm{~m}^{-2}$ current pulse with a time step of $2 \mathrm{~ns}$ [Fig. 2(b)]; and 6E11_POS_400ps.avi-Video5: Time-resolved STXM image (video) showing the CIDWM under the application of a $+6 \times 10^{11} \mathrm{~A} \mathrm{~m}^{-2}$ current pulse with a time step of 400 ps (Fig. 3). 
Part of this work was performed at the ptychography and STXM endstation of the Surface Interface Microscopy (X11MA) beamline of the Swiss Light Source, Paul Scherrer Institut, Villigen PSI, Switzerland. The research leading to these results received funding from the Swiss National Science Foundation, under Grant Agreement No. 172517, the EMPIR programme, under Grant No. 17FUN08TOPS, co-financed by the participating states, and from the European Union's Horizon 2020 research and innovation programme. M.L. acknowledges the funding received from the European Union's Horizon 2020 research and innovation programme under Marie Sklodowska-Curie Grant Agreement No. 701647.

The data that support the findings of this study are openly available in the Research Data Leeds Repository at https://www.doi.org/ $10.5518 / 808$.

\section{REFERENCES}

${ }^{1}$ D. Ralph and M. Stiles, J. Magn. Magn. Mater. 320, 1190 (2008).

${ }^{2}$ P. Gambardella and I. Miron, Philosoph. Trans. R. Soc. A 369, 3175 (2011).

${ }^{3}$ S. Parkin and S.-H. Yang, Nat. Nanotechnol. 10, 195 (2015).

${ }^{4}$ C. Garg, S.-H. Yang, T. Phung, A. Pushp, and S. Parkin, Sci. Adv. 3, e1602804 (2017).

${ }^{5}$ S.-H. Yang, K.-S. Ryu, and S. S. P. Parkin, Nat. Nanotechnol. 10, 221 (2015).
${ }^{6}$ M. Hayashi, L. Thomas, R. Moriya, C. Rettner, and S. Parkin, Science 320, 209 (2008).

${ }^{7}$ K.-S. Ryu, L. Thomas, S.-H. Yang, and S. Parkin, Nat. Nanotechnol. 8, 527 (2013).

${ }^{8}$ T. Taniguchi, K.-J. Kim, T. Tono, T. Moriyama, Y. Nakatani, and T. Ono, Appl. Phys. Express 8, 073008 (2015).

${ }^{9}$ R. Lo Conte, E. Martinez, A. Hrabec, A. Lamperti, T. Schulz, L. Nasi, L. Lazzarini, R. Mantovan, F. Maccherozzi, S. S. Dhesi, B. Ocker, C. H. Marrows, T. A. Moore, and M. Kläui, Phys. Rev. B 91, 014433 (2015).

${ }^{10}$ S. Finizio, S. Wintz, K. Zeissler, A. V. Sadovnikov, S. Mayr, S. A. Nikitov, C. H. Marrows, and J. Raabe, Nano Lett. 19, 375 (2019).

${ }^{11} \mathrm{~J}$. Vogel, M. Bonfim, N. Rougemaille, O. Boulle, I. M. Miron, S. Auffret, B. Rodmacq, G. Gaudin, J. C. Cezar, F. Sirotti, and S. Pizzini, Phys. Rev. Lett. 108, 247202 (2012).

${ }^{12}$ D. A. Allwood, G. Xiong, C. C. Faulkner, D. Atkinson, and R. P. Cowburn, Science 309, 1688 (2005).

${ }^{13}$ C. Garg, A. Pushp, S.-H. Yang, T. Phung, B. Hughes, C. Rettner, and S. Parkin, Nano Lett. 18, 1826 (2018).

${ }^{14}$ A. Puzic, T. Korhonen, B. Kalantari, J. Raabe, C. Quitmann, P. Jüllig, L. Bommer, D. Goll, G. Schütz, S. Wintz, T. Strache, M. Körner, D. Marko, C. Bunce, and J. Fassbender, Synchrotron Radiat. News 23, 26 (2010).

${ }^{15}$ U. Flechsig, F. Nolting, A. Fraile Rodriguez, J. Kremspasky, C. Quitmann, T. Schmidt, S. Spielmann, and D. Zimoch, AIP Conf. Proc. 1234, 319 (2010).

${ }^{16}$ M. Langer, C. A. F. Vaz, S. Chiriotti, A. Bergamaschi, M. Guizar-Sicarios, A. Kleibert, and J. Raabe, Microsc. Microanal. 24, 56 (2018).

${ }^{17}$ S. Finizio, K. Zeissler, S. Wintz, S. Mayr, T. Weßels, A. J. Huxtable, G. Burnell, C. H. Marrows, and J. Raabe, Nano Lett. 19, 7246 (2019). 